\title{
Allocation of Ventilators in a Public Health Disaster
}

\author{
Tia Powell, MD, Kelly C. Christ, MHS, and Guthrie S. Birkhead, MD, MPH
}

\section{ABSTRACT}

Background: In a public health emergency, many more patients could require mechanical ventilators than can be accommodated.

Methods: To plan for such a crisis, the New York State Department of Health and the New York State Task Force on Life and the Law convened a workgroup to develop ethical and clinical guidelines for ventilator triage.

Results: The workgroup crafted an ethical framework including the following components: duty to care, duty to steward resources, duty to plan, distributive justice, and transparency. Incorporating the ethical framework, the clinical guidelines propose both withholding and withdrawing ventilators from patients with the highest probability of mortality to benefit patients with the highest likelihood of survival. Triage scores derive from the sepsis-related organ failure assessment system, which assigns points based on function in 6 basic medical domains. Triage may not be implemented by a facility without clear permission from public health authorities.

Conclusions: New York State released the draft guidelines for public comment, allowing for revision to reflect both community values and medical innovation. This ventilator triage system represents a radical shift from ordinary standards of care, and may serve as a model for allocating other scarce resources in disasters. (Disaster Med Public Health Preparedness. 2008;2:20-26)

Key Words: ventilator, triage, guideline, influenza, pandemic

$\mathrm{I}$ $\mathrm{n}$ an overwhelming public health emergency, many more patients could require the use of mechanical ventilators than can be accommodated. Federal and state ventilator stockpiles exist, but in a disaster on the scale of the 1918 influenza pandemic, stockpiles would not be sufficient to meet need. Even if the vast number of ventilators needed for such a disaster were purchased, an insufficient number of trained staff would be available to operate the ventilators and care for critically ill patients, and access to ventilators would need to be rationed. To plan for such a crisis, the New York State Department of Health (NYSDOH) and the New York State Task Force on Life and the Law organized a workgroup to draft a set of ethical and clinical guidelines for the allocation of ventilators in an influenza pandemic. This article summarizes the development and content of the guidelines, the first of their kind in the United States, in the hope that they may serve as a template for rationing critical care resources in other public health disasters.

\section{METHODS}

In March 2006 the New York State Workgroup on Ventilator Allocation in an Influenza Pandemic (workgroup members are listed at the end of the article) brought together experts in medicine, policymaking, law, and ethics with representatives from medical facilities and city, county, and state government to address necessary alterations in the standard of care in an emergency. The workgroup met once and deliberated on key points until a general, although not always unanimous, consensus was reached. The draft document was written by task force staff and circulated in several iterations to workgroup members for editing and comment.

The workgroup developed an ethical framework for the allocation of ventilators during a pandemic. The workgroup then used this framework to derive an ethically and clinically sound set of guidelines for ventilator allocation. The draft guidelines were posted on the NYSDOH Web site on March 15, 2007, with a request for public comment. ${ }^{1}$ The guidelines have also been presented publicly, including at medical centers across New York state. Subcommittees of the workgroup focusing on critical care and legal issues were created to assess public comments. A revised version of the guidelines will be posted on the NYSDOH Web site when they become available.

\section{RESULTS}

Ethical Framework for Allocating Ventilators The workgroup began with the central concept that ethics cannot be set aside during a public health disaster. Rather, it is even more important in a crisis to articulate ethical norms for extraordinary circumstances and avoid the denigration of professional 
standards by decision makers under duress. The workgroup began its work by crafting an ethical framework for allocating ventilators in a public health emergency, incorporating the following elements:

- Duty to care

- Duty to steward resources

- Duty to plan

- Distributive justice

- Transparency

Duty to Care

An ethically sound rationing system must sustain the fundamental obligation of providers to care for patients. Physicians must not abandon, and patients should not fear abandonment, in a just system of allocation. Patients who are not eligible to receive mechanical ventilation will receive available forms of curative and palliative treatment.

Patient preference is not and cannot be the primary factor in devising a rationing system for ventilators because more patients will want ventilators than can be accommodated. A public health disaster, by virtue of severe resource scarcity, will impose harsh limits on decision-making autonomy for both patients and providers.

\section{Duty to Steward Resources}

The next element in the ethical framework is the obligation of government and health care providers to steward resources during a period of true scarcity. Balancing an obligation to the community of patients against the primary duty to care for each patient generates ethical tension in devising a rationing system. Clinicians need to save the greatest possible number of lives while continuing to care for each individual patient. As the number of affected patients multiplies, accommodating these 2 goals will require making increasingly difficult decisions.

\section{Duty to Plan}

An absence of guidelines leaves allocation decisions to exhausted frontline providers, who already bear a disproportionate burden in a disaster. A failure to produce acceptable guidelines for a foreseeable crisis amounts to a failure of responsibility toward both patients and providers. Health care providers at many of the presentations regarding the guidelines expressed concern about the arrest of several health professionals who worked during and after Hurricane Katrina. ${ }^{2,3}$ Appropriate guidance may help prevent both the actuality and the fear of similar consequences for those providing care in future emergencies.

Although planning is obligatory, any guidelines devised will be imperfect, both ethically and medically. Ethically, access to health care is unequal, and no rationing system for a crisis can resolve inequities in preexisting health status that result from unequal access. Medically, the clinical parameters of a pandemic are uncertain, increasing the difficulty of predicting benefit or survival. Despite the difficulties inherent in planning, public health entities must accept this responsibility.

\section{Distributive Justice}

To be fair, an allocation system must be applied broadly and consistently. The same allocation system should be used by all of the acute care facilities in the state. The decision to implement rationing at any facility must be triggered in collaboration with public health authorities and must be coordinated within the community, among communities, and between the local communities and the state. Cooperative agreements to pool scarce resources among local hospitals may help alleviate shortages. The allocation of ventilators from state and federal stockpiles must take into account the ratio of local populations to available resources and supplement those resources accordingly. Disparities in access to care and in health outcomes are highly significant in a state that is as diverse as New York. Ethically sound responses to disaster must not exacerbate such disparities. Rather, planners must designate appropriate resources for the most vulnerable, who are the most likely to experience the greatest impact in any disaster.

\section{Transparency}

A just system of allocating scarce resources requires transparency. The state should publicize proposed guidelines, translate them into different languages as necessary, share them with health care leaders and the community, including historically underserved communities, and seek public comment. Proposed revisions that will ensure a just allocation process should be incorporated.

Taking into account this ethical framework, guidelines for an allocation system for ventilators emerged. These draft guidelines propose both withholding and withdrawing ventilators from patients with the highest probability of mortality to benefit patients with a high likelihood of survival. Nonetheless, the workgroup struggled with the notion of extubating patients, even those unlikely to survive, to offer ventilators to those more likely to survive.

Ethicists in the workgroup argued that guidelines for decision making under duress are more likely to be followed when they seek to reduce the number of times that one confronts the most difficult decision. These guidelines permit patient extubation but aim to limit the times that clinicians face this most ethically and emotionally challenging aspect of the ventilator rationing system.

\section{Clinical Protocol}

The draft guidelines include the following ethically acceptable protocol for allocating ventilators in a public health emergency:

- Pretriage requirements

- Patient categories

- Acute versus chronic care facilities

- Clinical evaluation 
- Triage decision makers

- Palliative care

- Review of triage decisions

- Communication

\section{Pretriage Requirements}

Before rationing procedures are implemented, facilities should institute all available means of creating surge capacity. Hospitals should limit the noncritical use of ventilators, and elective procedures should be canceled and/or postponed. Facility, commercial, state, and federal ventilator stockpiles should be assessed, and additional ventilators (eg, transport or recovery room units) should be put to use. In a manageable crisis, instituting surge capacity measures may eliminate the need for rationing.

Facilities will need to document implementation of surge measures, working in collaboration with public health authorities, before they can access government ventilator stocks or institute rationing. The proposed allocation system represents a radical shift from ordinary standards of care. Triage may not be implemented by a facility without clear sanction from appropriate public health authorities.

Systems for sharing information about the number and severity of cases, equipment availability, and staffing shortages could be activated throughout hospital groups and regional networks. For instance, not all facilities may be equipped to care for infants who need ventilatory support. Clinicians and families will need rapid access to information about the location of such support.

People are the most valuable resource of any institution, including within health care facilities. Surge capacity must include securing adequate staff to operate ventilators and provide critical care. However, creating staff surge capacity presents a substantial challenge. In a pandemic, staff numbers will decrease as providers become ill, leave work to care for family, or decline to serve for fear of contagion, while the number of patients reaches unprecedented levels.

\section{Patient Categories}

A just rationing system must be applied to all hospitalized patients who require critical care, and not only to patients with influenza. As a practical matter, clinicians cannot limit the use of triage criteria to patients with any single diagnosis because critically ill patients may have multiple diagnoses or no clear diagnosis. Furthermore, a system that suggests a preference for one disease over others invites inaccurate reporting of diagnoses and could increase the danger of contagion.

The option of offering enhanced access to ventilators to health care providers, first responders, or other special groups sparks controversy. The workgroup participants, although not unanimously, propose that patients be assessed on medical/ clinical factors alone, regardless of their work role, for the reasons enumerated below.
First, health care workers who are sick enough to require ventilators are unlikely to regain their health and return to work during the pandemic. Mortality will be high even with ventilatory support, and the recovery period could be months. The worst phase of a pandemic will likely end before a stricken individual can return to work. Second, workers in many occupations risk exposure and provide crucial services in a pandemic. Doctors and nurses face risks, but so do respiratory therapists, janitors, morgue workers, laundry workers, ambulance staff, security personnel, firefighters, police officers, and others. Furthermore, it is not always easy to determine who is and who is not a health care worker. Part-time volunteers staff ambulances in some communities; an unpaid family member may serve as the full-time caregiver for a disabled relative. These unpaid providers take risks comparable to or greater than some paid health care providers. Expanding the category of privilege to include all of the workers listed above may mean that only health care providers obtain access to ventilators in certain communities. All other community members, including all children, could be denied access, a plan that was unacceptable to most of the workgroup. Because the majority of human fatalities attributable to the $\mathrm{H} 5 \mathrm{~N} 1$ avian influenza virus are in previously healthy children, ensuring access for children to scarce resources is particularly crucial in an influenza pandemic. ${ }^{4}$ Finally, workgroup members objected to the appearance of favoritism, in which those who devised the rationing system reserved special access for themselves.

The draft guidelines support access to ventilators based on clinical factors only. Of note, the allocation of other scarce resources, such as vaccine or antiviral medications, may well favor health care providers based on differing ethical and clinical considerations. ${ }^{5}$ Indeed, the stockpiling of personal protective equipment, including masks and gloves, is a crucial obligation for facilities and a means of protecting the health of professionals who take risks by working during a disaster.

\section{Acute Versus Chronic Care Facilities}

Patients using ventilators in chronic care facilities would not be subjected to acute care triage guidelines. If, however, such patients required transfer to an acute care facility, they would be assessed by the same criteria as all of the other patients, and may lose access to continued ventilator use. Chronically ill patients are especially vulnerable in public health emergencies. Chronic care facilities will have to provide more intensive care on-site as part of the general process of expanding care beyond standard locations. Barriers to transfer are appropriate and likely during a public health crisis.

An alternative approach would require assessing all of the intubated patients, whether in acute or chronic care facilities, by the same set of clinical criteria. Depending on the design of these criteria, the result may be the sudden and fatal extubation of stable, long-term ventilator-dependent patients in chronic care facilities. The proposed justification for such a strategy would be that more patients could ultimately 
survive if these ventilators were used by the previously healthy victims of a pandemic. However, this strategy would make victims of people with disabilities. More patients may survive, but they would also be different kinds of survivors. Such a strategy relies upon ethically unsound judgments based on third-party assessments of quality of life.

Applying acute care triage guidelines to chronic care facilities fails to adhere to the ethical principle of providing care for each patient, including the most vulnerable. The second principle of stewarding resources must also be considered. Setting aside the small number of ventilators in chronic care facilities for use by chronically ill people, who likely will have severely limited access to ventilators in acute care facilities, offers an appropriate balance between the duty to care and the duty to allocate wisely.

Small but increasing numbers of people who depend on mechanical ventilators reside in the community, rather than in institutions. Providing care for this group during a disaster poses significant challenges and should be the focus of additional planning efforts. In the absence of other specific provisions, workgroup participants concurred that communitydwelling individuals should not lose access to their ventilators.

\section{Clinical Evaluation}

A clinical evaluation system based on the Ontario Health Plan for an Influenza Pandemic (OHPIP) protocol and on the sequential organ failure assessment (SOFA) score is used in the draft guidelines. ${ }^{6-8}$ Incoming patients with clinical evidence of impending pulmonary failure meet the inclusion criteria and will be assessed for exclusion criteria, and then placed in categories based on a variation of the OHPIP system. Patients on ventilators when triage begins will also be assessed to determine whether they meet criteria for continued use. Candidates for extubation during a pandemic would include patients with the highest probability of mortality.

Exclusion Criteria Clinicians will assess patients for exclusion criteria. Patients who meet exclusion criteria will not have access to ventilators and will not enter into the scoring system (Table 1). A model set of exclusion criteria would define objectively those patients with a high risk for mortality even with ventilator support and would not rely on subjective judgments of quality of life. Exclusion criteria should focus primarily on current organ function rather than on specific diseases.

Age is not an exclusion criterion, and some question its omission from the draft guidelines. In particular, much public comment argued that it is more appropriate to maximize life-years saved rather than lives, a system that enhances access for children at the expense of older adults. Although age factors indirectly into any assessment of overall health, because chronic disease generally increases with age, the draft

\section{TABLE}

\section{Exclusion Criteria for Ventilator Access*}

Cardiac arrest

Unwitnessed arrest

Recurrent arrest

Arrest unresponsive to standard measures

Trauma-related arrest

Metastatic malignancy with poor prognosis

Severe burn: body surface area $>40 \%$, severe inhalation injury

End-stage organ failure

Cardiac: New York Heart Association class III or IV

Pulmonary: severe chronic lung disease with $\mathrm{FEV}_{1}{ }^{\dagger}<25 \%$

Hepatic: MELD ${ }^{\ddagger}$ score $>20$

Renal: dialysis dependent

Neurological: severe, irreversible neurological event/condition with high expected mortality

*Adapted from Ontario Health Plan for an Influenza Pandemic guidelines.

${ }^{\dagger}$ Forced expiratory volume in 1 second, a measure of lung function.

${ }^{\ddagger}$ Model of end-stage liver disease.

guidelines attempt to measure level of function rather than relying on age per se.

In contrast, renal failure is an exclusion criterion, and this, too, has elicited public comment. Renal failure increases morbidity and mortality in the intensive care unit, ${ }^{9}$ and dialysis places increased demand on scarce nursing resources. If additional comments emerge during the current phase of public engagement, then this or other exclusion criteria are subject to revision.

Initial Assessment There is no clinical scoring system that could supply quick, resource-sparing, reliable assessments of mortality in the event of the influenza pandemic considered by the workgroup. Triage systems used by the military assess trauma in otherwise healthy young people and so are not appropriate for scoring infectious disease in the general population. The SOFA scoring system has several imperfections - for instance, it has not been validated in childrenbut it remains the best available system. The SOFA score adds points based on objective measures of function in 6 domains: lungs, liver, brain, kidneys, blood clotting, and blood pressure (Table 2). A perfect SOFA score is 0 . The worst possible score is 24 , which indicates life-threatening abnormalities in all 6 systems.

The OHPIP protocol assigns patients to initial triage categories based on the criteria listed in Table 3 .

Time Trials Continued use of the ventilator will be reassessed at intervals of 48 and 120 hours. ${ }^{1}$ Patients showing improvement would continue ventilator use until the next assessment, whereas those who no longer met the criteria would lose access to mechanical ventilation. Time trials for ventilator use should reflect the expected duration of treatment for severe pulmonary complications. Excessively brief trials, such as only 24 hours, may allow the use of ventilators by more patients, but without decreasing overall mortality. 


\section{TABIE 2}

\section{Sequential Organ Failure Assessment (SOFA) Score*}

\begin{tabular}{|c|c|c|c|c|c|}
\hline Variable & 0 & 1 & 2 & 3 & 4 \\
\hline $\mathrm{PaO}_{2} / \mathrm{FiO}_{2} \mathrm{mmHg}$ & $>400$ & $\leq 400$ & $\leq 300$ & $\leq 200$ & $\leq 100$ \\
\hline Platelets, $\times 10^{3} / \mu \mathrm{L}\left(\times 10^{6} / \mathrm{L}\right)$ & $>150(>150)$ & $\leq 150(\leq 150)$ & $\leq 100(\leq 100)$ & $\leq 50(\leq 50)$ & $\leq 20(\leq 20)$ \\
\hline Bilirubin, mg/dL ( $\mu \mathrm{mol} / \mathrm{L})$ & $<1.2(<20)$ & $1.2-1.9(20-32)$ & $2.0-5.9(33-100)$ & $6.0-11.9(101-203)$ & $>12(>203)$ \\
\hline Hypotension & None & MABP <70 mmHg & Dop $\leq 5$ & $\begin{array}{c}\text { Dop }>5 \text {, Epi } \leq 0.1 \\
\text { Norepi } \leq 0.1\end{array}$ & $\begin{array}{l}\text { Dop }>15 \text {, Epi }>0.1 \\
\quad \text { Norepi }>0.1\end{array}$ \\
\hline Glasgow Coma Score & 15 & $13-14$ & $10-12$ & $6-9$ & $<6$ \\
\hline Creatinine, mg/dL ( $\mu \mathrm{mol} / \mathrm{L})$ & $<1.2(<106)$ & $1.2-1.9(106-168)$ & $2.0-3.4(169-300)$ & $3.5-4.9(301-433)$ & $>5(>434)$ \\
\hline
\end{tabular}

Dop indicates Dopamine; Epi, epinephrine; Norepi, norepinephrine. Doses in micrograms per kilogram per minute; SI units in parentheses.

*Data adapted from JAMA. ${ }^{8}$

Very short trials would also require terminal extubation for large numbers of patients, a circumstance that the guidelines should attempt to minimize.

\section{Triage Decision Makers}

Clinicians treating a patient will have neither the main nor the sole responsibility for making triage decisions. Clinicians providing direct care will relay data to a supervising clinician serving as a triage officer, who will calculate the SOFA score and make triage decisions but will not provide direct care. The triage officer will have information about the number and nature of patients awaiting admission to the unit and will set goals accordingly.

The criteria are intended to be simple and objective to apply, but the complexity of clinical circumstances will make actual triage decisions challenging. Of far greater importance than technical considerations, triage decisions will be difficult because of the impact on human lives. Guidelines for triage should minimize the erosion of clinicians' duty to care for individual patients. Establishing triage officers provides role sequestration that will help sustain clinicians who serve during disasters. Without such measures, the secondary effects of the disaster on clinicians, including burnout and stress, may prove more corrosive than the original trauma.

\section{Palliative Care}

When patients are extubated based on triage criteria, clinicians should follow existing facility protocols for withdrawing and withholding life-sustaining care and for providing pallia- tive care. Typically, terminal weaning in response to patient preferences can include sedation so that the patient need not experience air hunger. Patients who are extubated against their wishes should be offered appropriate palliative care based on their clinical conditions and preferences. Facility protocols for terminal extubation should offer guidance for appropriate dosing and procedures. Because transparency is a crucial element of adherence to ethical standards, clinicians must document decisions regarding sedation with extubation. Facilities should prepare for a significant increase in demand for expertise in palliative care. Extubated patients could receive nasal cannula oxygen if available or other breathing supplements.

Manual support for extubated patients using hand-held devices such as ambu-bags provides a low likelihood of benefit for patients and a high risk for volunteers. Family members and others who might provide such support face a high risk for infection. No individual can operate a manual device for long, and thus multiple volunteers would risk exposure in the likely futile attempt to help any single patient. In addition, a hand-held device does not provide the control of oxygen pressure and flow needed to sustain a critically ill patient over time. The guidelines do not support the use of manual ventilation devices for patients who do not meet criteria for ventilator access.

\section{Review of Triage Decisions}

Triage decisions will engender controversy and objections. A review process is needed to ensure consistency and justice in

\section{TABLE 3}

\section{Adapted OHPIP Triage Tool (Initial Assessment)}

Color Code

Blue

Red

Yellow

Green

\section{Criteria}

Exclusion criteria* or SOFA score $>11^{*}$

SOFA score $\leq 7$ or single organ failure

SOFA score 8-11

No significant organ failure
Priority/Action

High probability of mortality; should be discharged from critical care; medical management \pm palliate and $\mathrm{d} / \mathrm{c}$

Highest priority for critical care

Intermediate priority for critical care

Low probability of mortality; defer or $\mathrm{d} / \mathrm{c}$, reassess as needed

OHPIP indicates Ontario Health Plan for an Influenza Pandemic; d/c, discharge.

${ }^{*}$ If exclusion criteria or SOFA score $>11$ occurs at any time between the initial assessment to 48 hours, change triage code to blue and palliate. 
the application of the criteria, but a real-time appeals process could invite explosive debate during a time of scarce manpower and other resources. Daily retrospective review of all triage decisions is an alternative to a real-time appeals process. This review would ensure that standards are followed consistently and correctly and would present an opportunity for correcting the draft guidelines or their implementation as needed. Such retrospective review would provide oversight and accountability for triage decisions, but would not permit intervention for individual decisions.

\section{Communication}

Communicating information appropriately is one of the most significant challenges raised by a public health disaster. Physicians will need to discuss altered standards of care in a disaster, especially for scarce resources such as ventilators. Even before a patient comes to the hospital, political leaders and health officials must emphasize publicly that standards of care are and must be different in a public health disaster. Clinicians will do all that they can with the available resources, and the community will need to adjust to scarcity. Patients and families must be informed immediately that ventilator support represents a trial of therapy that may not improve the patient's condition sufficiently and that the ventilator will be removed if the patient does not meet specific criteria. Staff training for disaster readiness must include guidance on how to discuss such time trials. Communication must be clear upon hospital and intensive care unit admission, as well as upon initiation of ventilator treatment.

\section{Future Work}

Workgroup discussion and review regarding specific aspects of the guidelines continue, as does the process of soliciting public response. Review of the exclusion criteria and of portions of the clinical scoring system, especially regarding the assessment of children, need clarification. A subcommittee of critical care experts is reviewing the existing scoring system and will revise the guidelines as needed. An additional subcommittee will address the complex legal issues related to potential indemnification and liability of facilities and clinicians who follow the guidelines.

Finally, a series of focus groups across New York state are planned as a means of providing public education and soliciting comments from a range of community members, including parents, older adults, people with disabilities, and communities of color.

\section{CONCLUSIONS}

This article provides an overview of the development process and content of New York's guidelines for allocating ventilators in a public health crisis. Although the guidelines focus only on the allocation of ventilators, the process could serve as a template for the development of other policies regarding the allocation of scarce resources in public health emergencies. This allocation has life-and-death implications and touches upon community values of the utmost importance. Substantial efforts to engage the public in a discussion of these guidelines will help guarantee that allocation decisions reflect community values. The guidelines are structured to permit ongoing revision as needed to reflect both technical innovations and community values. These guidelines for allocating ventilators rely upon both ethical and clinical standards in an effort to offer the best possible care under gravely compromised conditions.

\section{New York State Workgroup on Ventilator Allocation in an Influenza Pandemic}

Co-Chairs: Guthrie S. Birkhead, MD; Tia Powell, MD

New York State Department of Health Representatives: Barbara Asheld, JD; Mary Ann Buckley, RN, MA, JD; Bob Burhans; Bruce Fage; Mary Ellen Hennessy, RN; Marilyn Kacic; John Morley, MD; Loretta Santilli; Perry Smith; Barbara Wallace, MD, MSPH; Dennis Whalen; Lisa Wickens, RN; Vicki Zeldin, MS

New York State Task Force on Life and the Law Staff: Michael Klein, JD; Kelly Christ, MHS

Outside Experts: Ron Bayer, PhD, Mailman School of Public Health Columbia University; Kenneth Berkowitz, MD, FCCP, New York University School of Medicine; Kathleen Boozang, JD, LLM, Seton Hall University School of Law; David Chong, MD, New York University School of Medicine; Brian Currie, MD, Montefiore Medical Center; Nancy Dubler, LLB, Montefiore Medical Center; Paul Edelson, MD, Mailman School of Public Health, Columbia University; Joan Facelle, MD, Rockland County Department of Health; Joseph J. Fins, MD, New York Presbyterian Hospital-Weill Cornell Center; Alan Fleischman, MD, New York Academy of Medicine; Lewis Goldfrank, MD, New York University School of Medicine; Patricia Hyland, MEd, RRT, RT, Hudson Valley Community College; Marci Layton, MD, New York City Department of Health and Mental Hygiene; Kathryn Meyer, JD, Continuum Health Partners Inc; Tom Murray, PhD, The Hastings Center; Margaret Parker, MD, FCCM, State University of New YorkStony Brook; Lewis Rubinson, MD, Centers for Disease Control and Prevention; Neil Schluger, MD, Columbia University College of Physicians and Surgeons; Christopher Smith, Healthcare Association of New York State; Kate Uraneck, MD, New York City Department of Health and Mental Hygiene; Susan Waltman, JD, MSW, Greater New York Hospital Association.

\section{About the Authors}

Dr Powell is Executive Director, New York State Task Force on Life $\mathcal{E}$ the Law; Ms Christ is World Trade Center Health Analyst, Division of Epidemiology, New York City Department of Health and Mental Health; and Dr Birkhead is Director, Center for Community Health, Director, AIDS Institute, New York State Department of Health.

Address correspondence and reprint requests to Dr Tia Powell, New York State Task Force on Life 89 the Law, 90 Church St, 15th Floor, New York, NY 10007 (e-mail: tpp03@health.state.ny.us).

Received for publication September 5, 2007; accepted November 12, 2007.

\section{Authors' Disclosure}

The authors report no conflicts of interest.

ISSN: 1935-7893 C 2008 by the American Medical Association and Lippincott Williams \& Wilkins.

DOI: 10.1097/DMP.0b013e3181620794 


\section{REFERENCES}

1. New York State Workgroup on Ventilator Allocation in an Influenza Pandemic. Allocation of Ventilators in an Influenza Pandemic: Planning Document, March 15, 2007. http://www.health.state.ny.us/diseases/communicable/influenza/pandemic/ventilators/docs/ventilator_guidance.pdf. Accessed August 24, 2007.

2. Curiel JT. Murder or mercy? Hurricane Katrina and the need for disaster training. New Engl J Med. 2006;355:2067-2069.

3. Drew C, Dewan S. Louisiana doctor said to have faced chaos. New York Times. July 20, 2006. http://www.nytimes.com/2006/07/20/us/ 20doctor.html?scp $=1 \&$ sq $=$ physician + arrested + after + Katrina + .

4. Writing Committee of the World Health Organization (WHO) Consultation on Human Influenza A/H5. Avian influenza A (H5N1) infection in humans. New Engl J Med. 2005;353:1374-1385.
5. Arras JD. Rationing vaccine during an avian influenza pandemic: why it won't be easy. Yale J Biol Med. 2005;78:287-300.

6. Christian MD, Hawryluck L, Wax RS, et al. Development of a triage protocol for critical care during an influenza pandemic. CMAJ. 2006;175: 1377-1381.

7. Ontario Health Plan for an Influenza Pandemic (OHPIP) Working Group on Adult Critical Care Admission, Discharge, and Triage Criteria. Critical Care During a Pandemic. April 2006. http://www.tufr.com/dept/em/ dnld/OHPIP-Critical_Car_\%20During_Pandemic-final-v2_0406.pdf. Accessed December 19, 2007.

8. Ferreira FL, Bota DP, Bross A, et al. Serial evaluation of the SOFA score to predict outcome in critically ill patients. JAMA. 2001;286:1754-1758.

9. Clermont G, Acker CG, Angus DC, et al. Renal failure in the ICU: comparison of the impact of acute renal failure and end-stage renal disease on ICU outcomes. Kidney Int. 2002;62:986-996. 